\title{
Comparison of the Efficacy of Melasma Treatments: A Network Meta-Analysis of Randomized Controlled Trials
}

\author{
Yi Liu ${ }^{1}$, Shanshan $W u^{2}$, Haixuan $W u^{1}$, Xuelei Liang ${ }^{1}$, Dechao Guo ${ }^{3}$ and Fenglin Zhuo ${ }^{1 *}$ \\ ${ }^{1}$ Department of Dermatology, Beijing Friendship Hospital, Capital Medical University, Beijing, China, ${ }^{2}$ National Clinical \\ Research Center of Digestive Diseases, Beijing Friendship Hospital, Capital Medical University, Beijing, China, ${ }^{3}$ Department \\ of General Surgery, The Third Affiliated Hospital of Zhejiang Chinese Medical University, Hangzhou, China
}

\section{OPEN ACCESS}

Edited by:

Salvador Gonzalez,

University of Alcalá, Spain

Reviewed by:

Francesco Lacarrubba,

University of Catania, Italy

Mauro Picardo,

San Gallicano Hospital, Italy

*Correspondence:

Fenglin Zhuo

zflsunny@hotmail.com

Specialty section:

This article was submitted to

Dermatology,

a section of the journal

Frontiers in Medicine

Received: 23 May 2021

Accepted: 20 August 2021

Published: 29 September 2021

Citation:

Liu Y, Wu S, Wu H, Liang X, Guo D and Zhuo $F$ (2021) Comparison of the

Efficacy of Melasma Treatments: A

Network Meta-Analysis of

Randomized Controlled Trials.

Front. Med. 8:713554.

doi: 10.3389/fmed.2021.713554
Background: Melasma is an acquired pigmentation disorder with challenges in treatment because of its refractory nature and high risk of recurrence.

Objectives: This study aimed to compare the efficacy and side effects of 14 common therapies for melasma using a systematic review and network meta-analysis (NMA).

Methods: The PubMed, Embase, and Cochrane Library databases were searched till December 2020 using the melasma area and severity index as a therapeutic index. A total of 59 randomized controlled trials (RCTs) met the inclusion criteria and were selected.

Results: The ranking of relative efficacy compared with placebo in descending order was Q-switched Nd:Yag 1,064-nm laser (QSND), intense pulsed light, ablative fractional laser (AFL), triple combined cream (TCC), topical vitamin C, oral tranexamic acid (OTA), peeling, azelaic acid, microneedles (MNs), topical tranexamic acid (tTA), tretinoin, picosecond laser, hydroquinone $(\mathrm{HQ})$, and non-AFL. Moreover, QSND was more effective than $\mathrm{HQ}$ and tTA against melasma. The ranking of percentage (\%) of side effects in ascending order for each of 14 therapies with more than 80 participants was tretinoin (10.1\%), oTA (17.6\%), HQ (18.2\%), AFL (20.0\%), QSND (21.5\%), TCC (25.7\%), tTA (36.75\%), peeling (38.0\%), and MN (52.3\%). Taking both efficacy and safety into consideration, TCC was found to be the most favorable selection among the topical drugs for melasma. QSND and AFL were still the best ways to treat melasma among photoelectric devices. OTA as system administration was a promising way recommended for melasma. Among 31 studies, $87 \%$ (27/31) studies showed that the efficacy of combination therapies is superior to that of single therapy. The quality of evidence in this study was generally high because of nearly $50 \%$ of split-face RCTs.

Conclusions: Based on the published studies, this NMA indicated that QSND, AFL, TCC, and OTA would be the preferred ways to treat melasma for dermatologists. However, more attention should be paid to the efficacy and safety simultaneously during the clinical application. Most of the results were in line with those of the previous studies, but a large number of RCTs should be included for validation or update.

Systematic Review Registration: identifier: CRD42021239203.

Keywords: melasma, efficacy, treatment, comparison, network meta-analysis 


\section{INTRODUCTION}

Melasma is a common and acquired skin disorder with pigmentation that predominantly affects women with darker skin types, such as Fitzpatrick skin types III and IV $(1,2)$. Melasma presents with bilateral, irregularly shaped, dark brown macules and usually appears on the cheeks, forehead, nose, or upper lip on the face $(3,4)$. The etiology of melasma is complicated; multiple factors, such as genetics, UV rays, pregnancy, and the use of hormonal contraceptives, maybe involved (5).

The treatment of melasma has been challenging because of its unclear etiology, stubbornly refractory nature, and frequent relapse. Besides, hyperpigmentation after treatment and long-term treatment makes it more difficult $(6,7)$. At present, the therapies of melasma can generally be divided into two categories: nonenergy-dependent and energydependent therapies. The former contains topical medicine such as hydroquinone (HQ), triple combined cream (TCC), azelaic acid (AA), tranexamic acid (TA), visible and UV light protection cream, chemical peelings, microneedles (MNs), platelet-rich plasma (PRP), ultrasound therapy, and so forth $(8,9)$. Nonenergy-dependent therapies, which have few risks of hyperpigmentation, take effect slowly. Energy-dependent therapies include intense pulsed light (IPL), Q-switched (QS) laser, picosecond laser (PICO), fractional laser, and so forth (10). Dark brown macules in patients with melasma were found to disappear quickly with energy-dependent therapies but easily relapsed or aggravated. Based on the literature review of the evidence, the first-line therapy was topical therapy, such as $\mathrm{HQ}$ cream and TCC, for at least 3 months. The second-line treatment indicated combination therapy with the first-line treatment and chemical peelings. The third-line therapy was the addition of laser and light-based devices to the aforementioned treatments (11). No single treatment that is particularly effective is available. Currently, combination therapies are preferable. Park et al. found that Q-switched Nd:Yag 1,064-nm laser (QSND) combined with glycolic acid (GA) had a beneficial effect on recalcitrant mixedtype melasma, with minimal side effects (12). Nouri et al. showed slightly higher efficacy of $\mathrm{CO}_{2}$ laser $+\mathrm{QS}$ alexandrite laser than $\mathrm{CO}_{2}$ laser alone (13).

Selecting the best combination therapies from a variety of treatments is worth exploring. In recent years, a larger number of randomized controlled trials (RCTs) were reported for melasma, but they were limited to comparisons between two or three treatment methods. Quantitative comparisons of the efficacy between energy-dependent and nonenergydependent therapies for melasma would be a valuable tool for dermatologists, significantly guiding treatment decisions and influencing patient outcomes. Although multiple treatments are available for melasma, no evidence-based ranking exists for it. In this study, the first network meta-analysis (NMA) of melasma treatments was conducted using 59 selected RCTs including 14 common interventions: nonenergy-dependent treatments (HQ, topical tranexamic acid (tTA), oral tranexamic acid (oTA), AA, TCC, peeling, topical vitamin $\mathrm{C}(\mathrm{tVC})$, tretinoin, and $\mathrm{MN})$ and energy-dependent treatments [IPL, QSND, PICO, ablative fractional laser (AFL), and nonablative fractional laser (NAFL)].
The efficacy of 14 therapies was directly and indirectly compared with placebo using the mean change of melasma area and severity index (MASI) before and after treatment as the outcome measure. This study aimed to provide an evidence-based comparison for treating melasma.

\section{MATERIALS AND METHODS}

This study is registered on the International Prospective Register of Systematic Review PROSPERO, number CRD42021239203.

\section{Databases and Search Strategy}

The databases PubMed, Embase, and Cochrane Library were searched for original studies, and the search ended on December 2020. The keywords used were "melasma" or "chloasma" in the title/abstract, and filters for "randomized controlled trial" and "controlled clinical trial" were applied to the search.

\section{Study Selection}

This systematic review and meta-analysis were conducted in agreement with the 2015 modified 32-item PRISMA extension statement for NMA (14). Only RCTs published in English were eligible for inclusion. RCTs could be placebo control studies or comparison trials of different therapies including single or combination therapy. Outcomes of interest were MASI and adverse events. MASI measures included evaluation of MASI, modified MASI (mMASI), or hemi-MASI. Some uncommon treatments for melasma were excluded, which included PRP, cysteamine, pulsed dye laser, and silymarin andrumex occidentalis.

\section{Data Extraction and Quality Assessment}

The following details were extracted: trial information (author, publication year, sample size, trial duration, and types of intervention), population characteristics (age and skin types), outcomes of efficacy (change in MASI), and adverse effects (events and numbers). The time regime with the biggest change in MASI was selected as assessment endpoints. The risk of bias among the included studies was assessed according to the Cochrane risk-of-bias (ROB) tool (15).

\section{Data Synthesis and Analysis}

\section{Methods for Direct Comparisons of Treatments}

DerSimonian and Laird random-effects model was used for standard paired meta-analysis. The standardized mean difference (SMD) of 95\% CI of each result was calculated as the efficacy index.

\section{Methods for Indirect and Mixed Comparisons}

The random-effects NMA was performed within the Bayesian framework (16). The SMD of each result with $95 \%$ CI was summarized. The ranking of treatments was summarized and reported as the surface under the cumulative ranking curve (SUCRA) and mean ranks. The SUCRA is a percentage interpreted as the probability of the most effective treatment. 


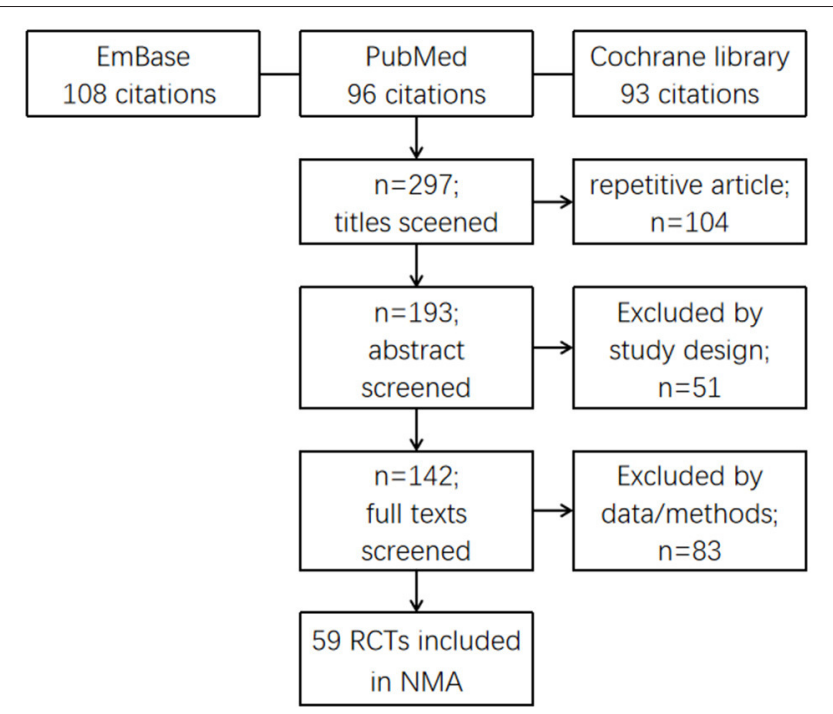

FIGURE 1 | Flow chart showing the screening process of the literature.

\section{Examination of Assumptions in NMA}

The mean difference (MD) was used to analyze the inconsistency between direct evidence and indirect evidence (17). A $P<0.05$ indicated a significant inconsistency between direct evidence and indirect evidence in the network.

STATA version 15.0 software (StataCorp LLC, 4905 Lakeway Drive, college station, TX 77845, USA) was used for data analysis, namely, pairwise meta-analysis, estimation of inconsistency, and forest plot. The ROB was performed using RevMan 5.3 (The Nordic Cochrane Centre, The Cochrane Collaboration, Copenhagen, Denmark).

\section{RESULTS}

\section{Results of the Search}

A total of 297 studies were identified by the literature search (PubMed, Embase, and Cochrane Libraries) (Figure 1). Only 59 studies met the inclusion criteria and were included in the quantitative NMA. Detailed information for all these studies presented in the NMA is shown in Supplementary Table 1.

The included trials had 2,812 randomized participants, of whom $7.1 \%$ were men. The average age of the participants was $39.25 \pm 6.69$ years, and the score of MASI at baseline was 11.34 \pm 4.51 . A total of 40 trials among 59 included studies clearly reported Fitzpatrick skin types as follows: IV $(n=451)$, III $(n$ $=353), \mathrm{V}(n=332)$, II $(n=92)$, and VI $(n=1)$. For the limited records of melasma type, the epidermal type $(n=280)$ was the most common type among the participants, followed by mixed type $(n=105)$ and dermal type $(n=57)$. Except for three studies that treated only one session, the average duration of treatment in the remaining studies was 12 weeks, ranging from 5 to 30 weeks. The final assessment endpoint was 4-40 weeks, with an average of 13 weeks; 26 studies involved single treatment and 33 involved combination therapy. Again, 25 of 59 studies were split-face
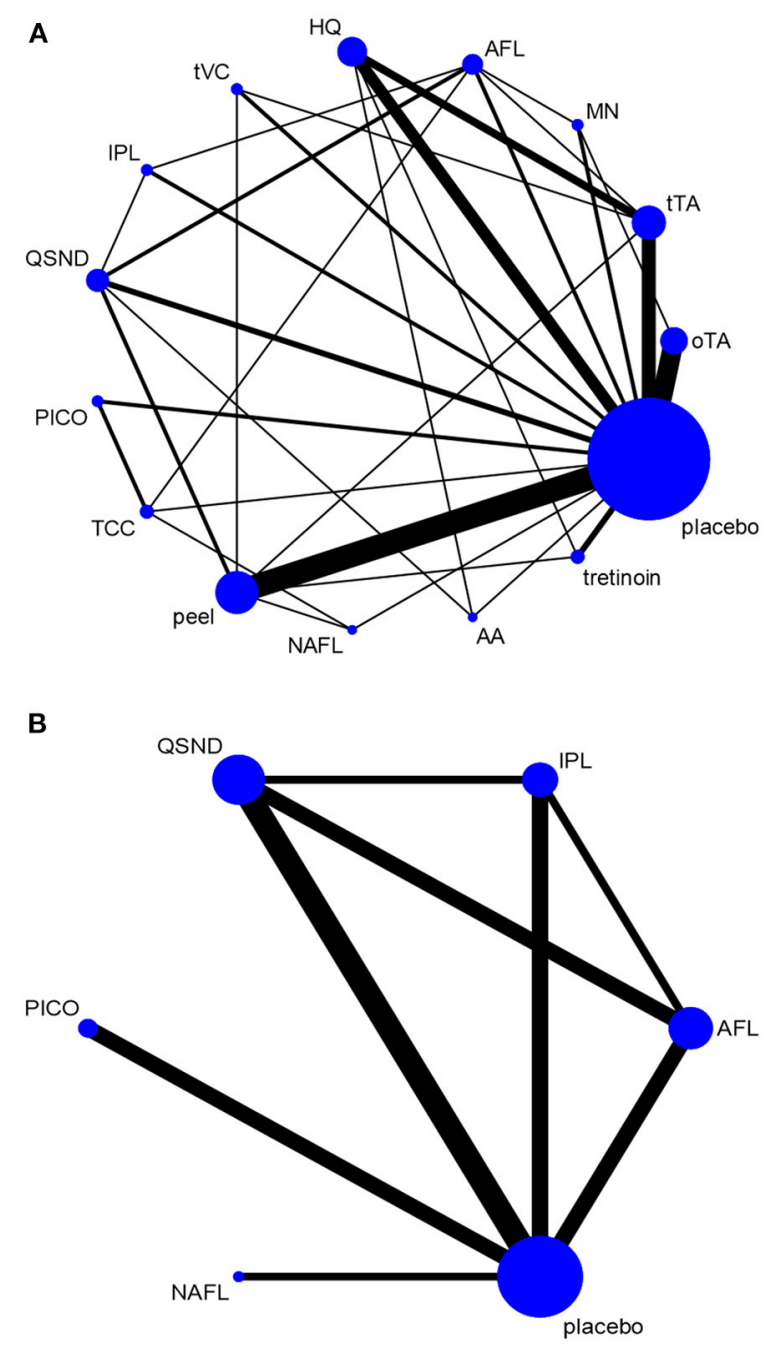

C

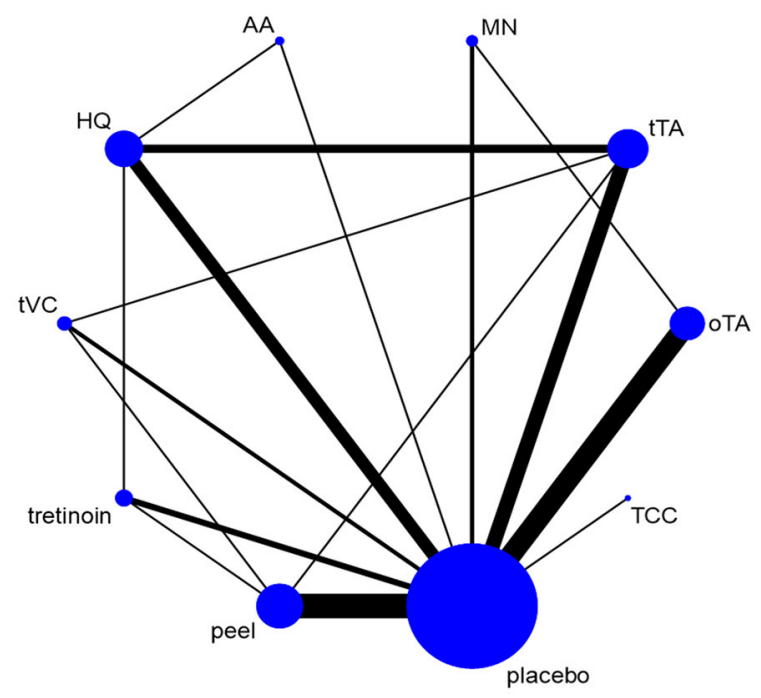

FIGURE 2 | Network graph. The network graph shows the evidence network for all selected interventions. (A) Network graph for all selected interventions; (B) network graph for energy-dependent treatments; and (C) network graph for nonenergy-dependent treatments. 
control studies, nine studies were performed in more than two treatment arms, and the rest were pairwise comparative studies.

The total network graph is provided in Figure 2A. The graph included placebo and 14 treatments, of which five therapies were energy dependent and nine were nonenergy dependent. Figure 2B shows the network graph of energy-dependent treatments compared with placebo. Figure $2 \mathrm{C}$ presents the graph of nonenergy-dependent treatments compared with placebo.

\section{Risk of Bias}

The ROB graph evaluated using the Cochrane Collaboration's tool is shown in Figures 3, 4. Generally, low-risk RCTs account for more than $80 \%$ in six domains except for one domain (performance bias), which is attributed to $37.5 \%$ (22/59) splitface trials and $5 \%(3 / 59)$ without special description. The random sequence generation (selection bias) in all studies (59/59, 100\%) was properly described; $94.9 \%$ (56 of 59) RCTs had a low risk of allocation concealment (selection bias), and $83.0 \%$ (49 of 59) had a low risk of detection bias. In addition, 6.7\% (4 of 59) and $8.4 \%$ ( 5 of 59) RCTs were unclear in reporting bias and other bias, separately. Overall, the ROB was low.

\section{Direct Pairwise Meta-Analysis and NMA Direct Pairwise Meta-Analysis}

The meta-analysis of direct pairwise comparisons (Figure 5A) showed that 14 treatments exhibited greater efficacy than placebo. The most effective treatment was TCC (MD [95\% CI]: $130.19[8.85,1,914.41])$, followed by tVC (MD [95\% CI]: 102.57 [7.76, 1,355.08]), AFL (MD [95\% CI]: 89.24 [12.32, 646.34]), and QSND (MD [95\% CI]: 86.32 [13.54, 550.40]); tretinoin (MD [95\% $\mathrm{CI}]: 6.20[0.62,62.17])$ was the worst. Direct comparisons for all treatments are shown in Figure 5B.

\section{Mixed Treatment Comparisons}

The results of the NMA comprising monotherapies and combination treatments are shown in Figure 6. It indicates that $64.3 \%$ of treatments ( 9 of 14) in the relative effects were superior to placebo. Additionally, QSND demonstrated greater efficacy over tTA and HQ $(2.75[5.12,0.39]$ and $16.615[0.45,5.47])$.

\section{Inconsistency Analysis}

The results from the node-splitting analysis of consistency/inconsistency comparisons are shown in Figure 7. The $P$ value was used to measure consistency by calculating the probability of observing the results from samples of data, assuming that the null hypothesis was true. The smaller the $P$ value, the greater the inconsistency. A $P<0.05$ indicated significant inconsistency between direct and indirect evidence in the network. In general, no significant difference was found (total $P=0.181$ ) in four out of the $33 P$ value measures; only two comparisons indicated significant differences.

\section{Ranking of Treatments by Efficacy}

The SUCRA was used as a probability to evaluate the efficacy of the treatment. SUCRA is a percentage representing the efficacy of each intervention compared with the placebo. It is used to provide the hierarchy of treatments and illustrate

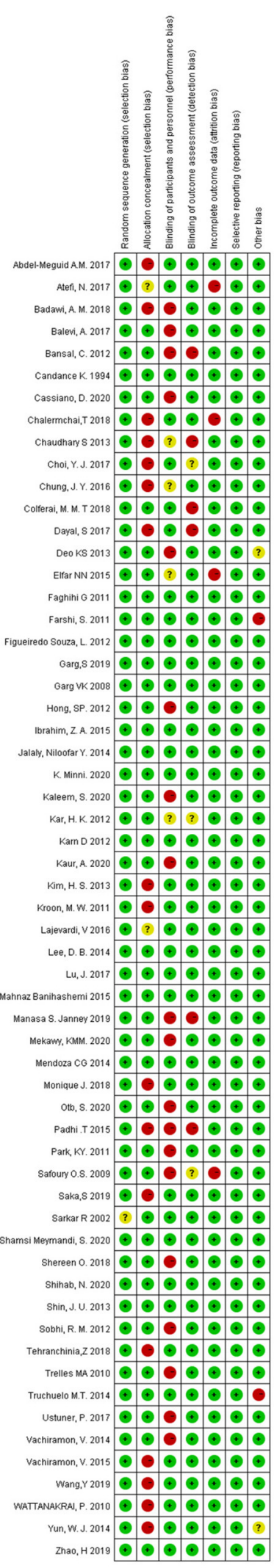

FIGURE 3 | Risk of bias summary for individual studies. 


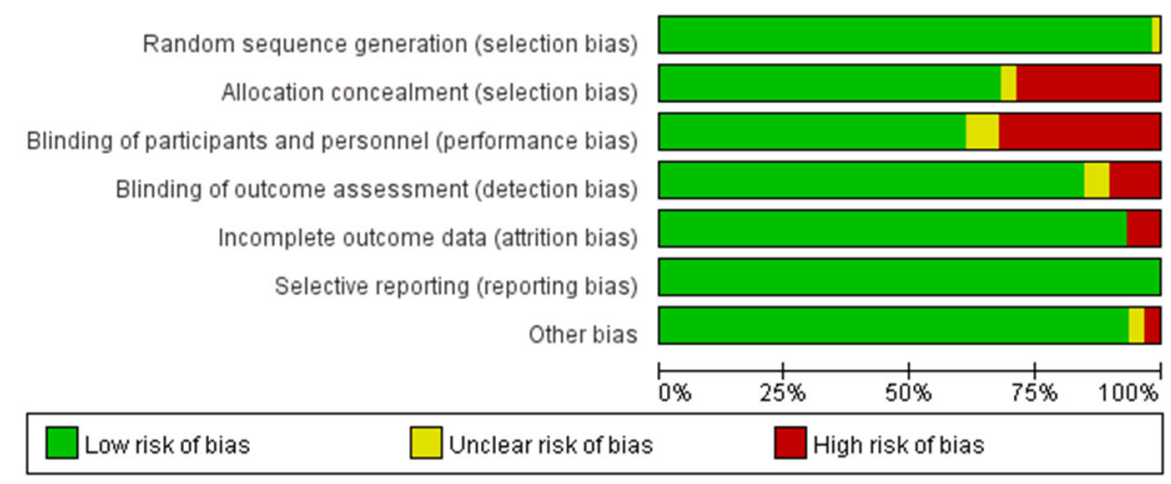

FIGURE 4 | Risk of bias graph: review judgments of authors about each risk of bias item presented as percentages across all included studies.

the location and variance of all relevant therapeutic effects. The higher the SUCRA score, the higher the probability of effectiveness.

The top six of SUCRA scores demonstrated QSND with the highest SUCRA (85.0\%), followed by IPL (79.6\%), AFL (78.6\%), TCC (75.8\%), tVC (68.5\%), and oTA (63.3\%). HQ and NAFL reported lower scores (29.4 and $28.1 \%$, respectively) (Table 1).

\section{Side Effects}

The summary of side effects for each monotherapy included in the NMA is listed in Table 2. The adverse effects involved burning or stinging, erythema, scaling, swelling, hyperpigmentation, edema, and so forth. Erythema and burning were the most common side effects. In this study, the participant numbers for NAFL (27), PICO (20), AA (64), tVC (31), and IPL (47) were too less to be statistically analyzed. Given each treatment with more than 80 participants was considered significant, the ranking of side effects with percentage $(n / N \%)$ in ascending order was tretinoin (10.19\%), oTA (17.65\%), HQ (18.20\%), AFL (20\%), QSND (21.5\%), TCC (25.775), tTA (36.75\%), peeling (38.03\%), and $\mathrm{MN}(52.33 \%)$. Owing to the limited reports of side effects in detail, the results were only for reference.

\section{DISCUSSION}

In recent years, the treatments of melasma received immense attention due to its refractory nature and high relapse risk. The NMA from 59 RCTs in this study compared the clinical efficacy of 14 common interventions for melasma with a mean change in MASI scores and percentage of side effects. The results showed that compared with placebo, local therapies (TCC, tTA, peeling, and HQ), systemic medication (oTA), and device-based treatments (QSND, IPL, AFL, and NAFL) demonstrated significantly greater odds ratios of MASI change. In addition, QSND showed significantly greater odds ratios of MASI decrease in comparison with tTA and HQ. Generally, the quality of evidence graded mostly moderate to high. Although two groups of direct and indirect comparison results existed, which showed inconsistency $(P<0.05)$, no inconsistency was observed on the whole $(P=0.1814)$.

The SUCRA ranking provided a summary of efficacy with 14 interventions that clinicians could refer to, but it had the potential to be misleading. Rankings need to consider the quality of evidence and the context of the main results of an NMA, which is the relative treatment effect. A treatment may rank favorably, but the low quality of evidence with the risks of bias, inconsistency, and/or imprecision may cast doubt on confidence in such a favorable ranking (18).

In recent years, energy-based devices are becoming popular treatments for melasma. Melanocytes and removal of dermal melanophages could be destroyed through subcellular selective photothermolysis (Figure 8) (19-21). In addition, AFL and NAFL could remodel the skin tissue to reverse antiphotoaging by creating micro-areas of thermal injury. However, the risk of melanocyte irritation and postinflammatory hyperpigmentation (PIH) cannot be ignored, especially the high risk of PIH after NAFL and AFL treatment, but this also increases the risk of side effects $(22,23)$. The laser treatment for melasma requires the optoelectronic theory, practical ability, and experience of the operating doctor, which should be carefully selected according to the specific conditions of the doctor and the patient. According to our results, energy-based devices have different effects on melasma.

The top three treatments of the SUCRA ranking in descending order were QSND, IPL, and AFL, which were all energydependent therapies. Definitely, large-spot and low-energy QSND has been widely used to treat melasma, and the curative effect is obvious (24-26). Abdel et al. confirmed the effectiveness of QSND for melasma by observing the changes in dermatoscopy and in vivo confocal microscopy $(27,28)$. IPL ranked second with only three studies, and IPL-based combination therapy was discussed in two studies. In a meta-analysis-based assessment, IPL-based combination therapies had a significantly lower MASI compared with the single-treatment group ( $\mathrm{SMD}=0.61, \mathrm{CI}$ $[0.42,0.80] P<0.0001)$ (29). This result was consistent with the current ranking. 


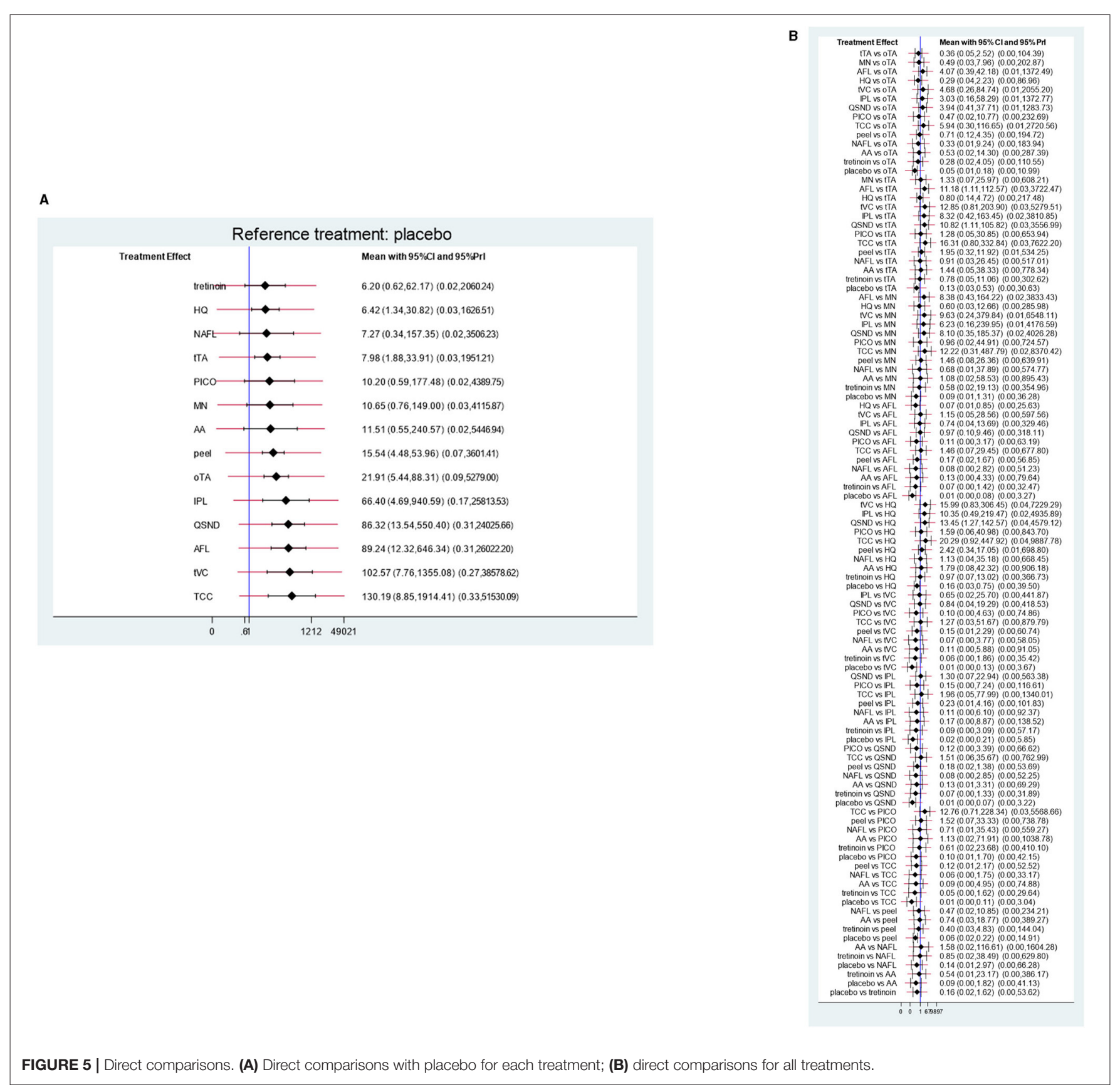

Ablative fractional laser applied in melasma included fractional $\mathrm{CO}_{2}$ laser and 2,940-nm Er:YAG laser. A single treatment with either fractional $\mathrm{CO}_{2}$ laser or 2,940-nm Er:YAG laser in two sessions with a 1-month interval reduced MASI by 48 and $26 \%$, respectively $(23,30)$. AFL combined with tTA or TCC also demonstrated higher efficacy on melasma $(31,32)$. PICOs, as a newly emerging treatment for melasma with only three published RCTs, ranked far behind QSND, IPL, and AFL in this study. This conclusion was inconsistent with a split-face study in which 755-nm PICO was more effective than QSND for melasma in Asians (33). Another study further proved that a significantly greater decrease in melanin-induced reflectance in the spinous layer and the basal layer was observed after $755 \mathrm{~nm}$ of PICO treatment compared with the QSND treatment for melasma (34). More RCTs with PICO for melasma should be included in the future.

Following QSND, IPL, and AFL, TCC ranked fourth in superiority to other nonenergy-dependent treatments, such as oTA, peeling, MN, and other topical agents, which should be inseparable from the combined efficacy of three pharmaceutical ingredients (Figure 8) (35). TCC consists of HQ, a retinoid, and a 


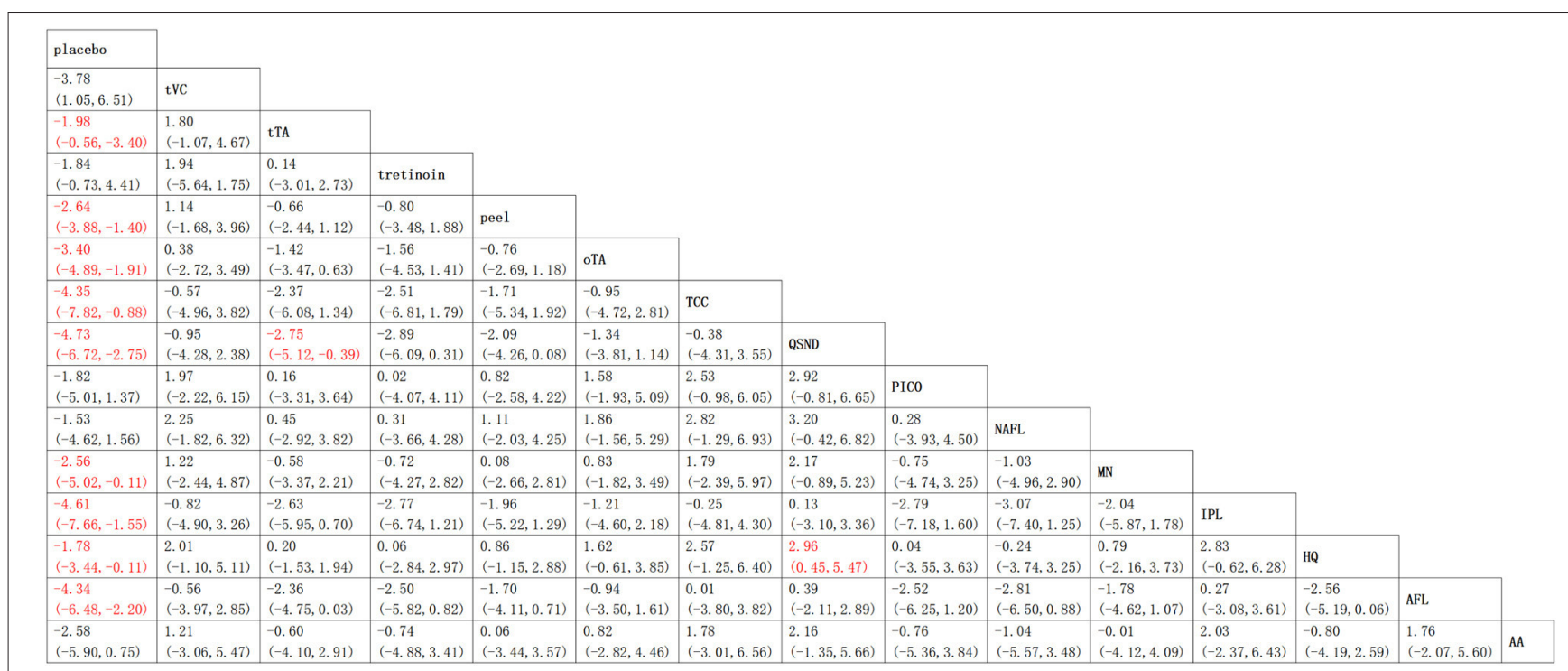

FIGURE 6 | Relative effects table. Comparison of the included interventions: mean difference (95\% credible intervals). Each cell gives the effect of the column-defining intervention relative to the row-defining intervention.

fluorinated corticosteroid and is widely regarded as the safest and effective treatment for melasma (36). In a large, multicenter RCT, TCC was found to be more effective than any dual combination of the three active ingredients (37). Studies showed that the efficacy of TCC in treating melasma was far better than that of HQ $(38,39)$ and was equivalent to a $755-n m$ PICO laser $(40)$. No direct evidence is available to compare TCC with other topical drugs. Except for TCC, the ranking of other topical agents in descending order was $\mathrm{tVC}, \mathrm{AA}, \mathrm{tTA}$, tretinoin, and HQ. Topical vitamin $\mathrm{C}$ (VC) and tTA rankings were inferior to HQ in this study, which might be relevant to the delivery mode with VC iontophoresis or intradermal TA (41). Besides that, the action of multiple targets in the therapeutic mechanism is also an important factor. VC can not only inhibit the transformation of tyrosine to pigment but also be antioxidant-promoting collagen synthesis (42). Antiphotoaging in the melasma area is recently considered to be an important strategy for melasma therapy. Energy-based devices, MN, peeling, TCC, etc. are also considered to remodel the aging microenvironment of melasma (Figure 8).

Tranexamic acid also showed superior effects on melasma in this study. TA for melasma targets melanocyte, endothelial cell, mast cell, and keratinocyte to remove pigmentation (Figure 8) (43, 44). Owing to the expected effect, oTA has been recommended as first-line therapy in a recent review (11). Recently, the interest has grown in studying the effects of TA in various formulations, namely, topical, intradermal, and oral, for melasma. In a meta-analysis, oTA demonstrated the greatest improvement compared with topical and intradermal formulations (45). However, more studies are needed to determine its long-term safety and efficacy. Apart from oTA, another oral agent on melasma, Polypodium leucotomos extract (PLE) is an antioxidant with photoprotective effect and immunoprotective activities UVA and UVB radiation. Till now, two RCT studies of PLE on melasma have been published. At the end of the two studies, there were no significant differences between oral PLE and placebo $(46,47)$. This conclusion should attribute to the single target to UV radiation protectant as an adjunct to topical sunscreen.

Chemical peeling is the recommended second-line treatment for melasma, and it can accelerate epidermal renewal and collagen regeneration to remove epidermal melanin and suspend the transfer of melanosomes (48). A systematic review and metaanalysis showed that GA peeling had no obvious advantage over tretinoin (MD 0.00, 95\% CI [0.99, 0.99], $P=0.3$ ) and VC (MD 1.50, 95\% CI [0.50, 3.50], $P=0.14$ ), while TCA peeling (MD 5.30, 95\% CI [6.41, 173 4.19], $P<$ 0.001 ) and Jessner solution (MD 3.20, 95\% CI [5.35, 1.05], $P=0.004)$ were better than HQ in treating melasma (49). Chemical peeling in this study included GA, TCA, SA, and Jessen solution. In the SUCRA ranking, peeling held the middle position (7/14), also ranking higher than tretinoin and HQ.

The role of microneedling in melasma ranked ninth in this study. MN creates minor skin damage, and in the process of skin self-repairment, it may reverse some structural patterns of melasma (50). Most studies assessed the use of $\mathrm{MN}$ in combination with other topical agents for the facilitation of drug delivery. Very few studies are available on MN monotherapy. Cassiano et al. (50) showed that a one-time treatment with dermaroller microneedling reduced mMASI by $9 \%$ after a 1 week follow-up, which was significantly more than that in the control group. Above all, the advantage of $\mathrm{MN}$ includes the very low risk of PIH. For the choice of melasma treatment, besides effectiveness, side effects are also an important factor. 


\begin{tabular}{|c|c|c|c|c|c|c|c|c|}
\hline & & \multicolumn{2}{|l|}{ Direct } & \multicolumn{2}{|l|}{ Indirect } & \multicolumn{3}{|l|}{ Difference } \\
\hline & & Coef. & Std. Err. & Coef. & Std. Err. & Coef. & Std. Err. & $\mathrm{P}>|\mathrm{z}|$ \\
\hline oTA & MN & -0.2000003 & 2. 422106 & -1.136464 & 1. 660675 & 0.9364635 & 2. 936739 & 0.75 \\
\hline oTA & placebo & -3.471177 & 0.7990324 & -2.549254 & 2. 753432 & -0.9219234 & 2.867085 & 0.748 \\
\hline $\mathrm{tTA}$ & $\mathrm{AFL}$ & -0.9399907 & 2. 321263 & 3. 54846 & 1. 397807 & -4.488451 & 2. 709633 & 0.098 \\
\hline tTA & $\mathrm{HQ}$ & -0.4101021 & 1. 209869 & 0.0448149 & 1. 327848 & -0.4549171 & 1. 79641 & 0.8 \\
\hline tTA & tVC & -0.4399965 & 2. 605773 & 2. 83924 & 1. 773637 & -3.279237 & 3. 152091 & 0.298 \\
\hline $\mathrm{tTA}$ & peel & 2. 199921 & 2. 448987 & 0.4123992 & 0.9825303 & 1. 787522 & 2. 638732 & 0.498 \\
\hline tTA & placebo & -1.263878 & 0.9389898 & -3.001888 & 1. 122823 & 1. 73801 & 1. 463681 & 0.235 \\
\hline MN & $\mathrm{AFL}$ & -0.1099995 & 2. 323424 & 2. 98595 & 1. 859085 & -3.09595 & 2. 975651 & 0.298 \\
\hline $\mathrm{MN}$ & placebo & -1.183658 & 1. 728844 & -4.077464 & 1. 812361 & 2. 893806 & 2.504955 & 0.248 \\
\hline AFL & IPL & -2.98933 & 3. 125661 & 1. 631841 & 2. 020382 & -4.621171 & 3. 721731 & 0.214 \\
\hline AFL & QSND & -4.539326 & 1. 953262 & 3. 44889 & 1. 49715 & -7.988216 & 2. 462333 & 0.001 \\
\hline AFL & $\mathrm{TCC}$ & 2. 799687 & 3. 719697 & -1.04087 & 2. 28449 & 3. 840557 & 4. 365073 & 0.379 \\
\hline AFL & placebo & -2.977211 & 2. 037898 & -4.9009 & 1. 304041 & 1.923689 & 2. 420381 & 0.427 \\
\hline $\mathrm{HQ}$ & $\mathrm{AA}$ & 2. 799758 & 2. 593041 & -0.8110114 & 2. 327034 & 3. 610769 & 3. 484056 & 0.3 \\
\hline $\mathrm{HQ}$ & tretinoin & -1.440226 & 3. 319408 & 0. 4395521 & 1. 664002 & -1.879778 & 3. 713087 & 0.613 \\
\hline $\mathrm{HQ}$ & placebo & -2.179162 & 1. 158185 & -1.301769 & 1. 258055 & -0.8773927 & 1. 710052 & 0.608 \\
\hline tVC & peel & -1.776269 & 2. 518938 & -0.8360121 & 1. 771252 & -0.9402571 & 3. 079367 & 0.76 \\
\hline $\mathrm{tVC}$ & placebo & -4.781437 & 2. 091796 & -2.972533 & 1. 889768 & -1.808904 & 2. 819707 & 0.521 \\
\hline IPL & QSND & 0.090145 & 2. 772834 & 0.1464949 & 2. 065816 & -0.0563499 & 3. 457813 & 0.987 \\
\hline IPL & placebo & -6.13437 & 2. 166277 & -2.962999 & 2. 244977 & -3.171371 & 3. 118365 & 0.309 \\
\hline QSND & peel & 0.1242381 & 2. 030616 & -3.01518 & 1. 305349 & 3. 139418 & 2. 414604 & 0.194 \\
\hline QSND & $\mathrm{AA}$ & -4.779534 & 2. 857945 & -0.4740979 & 2. 290676 & -4.305436 & 3. 662594 & 0.24 \\
\hline QSND & placebo & -7.936233 & 1. 417001 & -2.226802 & 1. 278096 & -5.709431 & 1. 909241 & 0.003 \\
\hline PICO & TCC & 1. 803406 & 2. 28098 & 3. 729765 & 2. 916761 & -1.926359 & 3. 70271 & 0.603 \\
\hline PICO & placebo & -1.308985 & 1. 903616 & -3.23719 & 3. 173464 & 1.928206 & 3. 700321 & 0.602 \\
\hline $\mathrm{TCC}$ & NAFL & -1.499878 & 3. 500666 & -3.564115 & 2. 630742 & 2. 064237 & 4. 379019 & 0.637 \\
\hline TCC & placebo & -4.9995 & 3. 699686 & -4.160736 & 2. 020898 & -0.8387639 & 4. 215853 & 0.842 \\
\hline peel & NAFL & -0.0500722 & 2. 483332 & -1.869787 & 2. 114208 & 1. 819715 & 3. 261388 & 0.577 \\
\hline peel & tretinoin & 0.2999729 & 2. 374277 & -1.357782 & 1. 684839 & 1.657755 & 2. 911329 & 0.569 \\
\hline peel & placebo & -2.46755 & 0.743648 & -3.124835 & 1. 244308 & 0.6572844 & 1. 449904 & 0.65 \\
\hline NAFL & placebo & -0.0300128 & 2. 316313 & -2.842133 & 2. 161433 & 2. 81212 & 3. 168142 & 0.375 \\
\hline $\mathrm{AA}$ & placebo & -2.79016 & 3. 075251 & -2.47588 & 2. 05084 & -0.3142797 & 3. 696483 & 0.932 \\
\hline tretinoin & placebo & -1.655119 & 1. 747995 & -2.07836 & 2. 00736 & 0.4232411 & 2. 662181 & 0.874 \\
\hline
\end{tabular}

FIGURE 7 | Results from the node-splitting analysis of consistency/inconsistency comparisons.

The side effects involved in published studies for 14 therapies were summarized in this study. Generally speaking, oTA, with the fifth position in relative efficacy and second position in side effects (17.6\%), is a promising and preferred way for melasma therapy. This conclusion was following other studies $(45,51)$. Photoelectric devices, such as QSND and AFL, ranked first and third in efficacy, show similar side effects (21.5 and 20\%) but a little higher than those of oTA (17.65\%). In consequence, QSND and AFL are worthy of being applied as a monotherapy or combination therapy for melasma. Among topical medicines, single tretinoin or HQ is not recommended as the first choice owing to their low efficacy. We recommend HQ combined with photoelectric devices for melasma. TCC, ranking fourth in efficacy, is a favorable selection with moderate side effects $(22.7 \%)$ for melasma. Peeling and MN with a high incidence of side effects in this study were not the preferable methods so far. Therefore, besides ensuring the effectiveness of treatment, the side effects after treatment should also be considered and appropriate posttreatment care should be provided to reduce the occurrence of melasma.

Neagu et al. (52) suggested that no single treatment for melasma is generally effective. Compared with a single treatment, both double and triple treatments show good curative effects (52). Among 56 included studies in the study, 31 studies compared the efficacy of combination therapy with a single therapy, of which 27 considered that the efficacy of combination therapy was better than that of single therapy, and four considered 
that there was no significant difference between the two. In general, the efficacy of interventions with multitarget effect in the treatment of melasma is better than those with a single target.

TABLE 1 | Ranking of competing treatments included in the network for melisma.

\begin{tabular}{lccc}
\hline Treatment & SUCRA $^{*}$ & PrBest & MeanRank \\
\hline QSND & 85.0 & 19.7 & 3.1 \\
IPL & 79.6 & 26.4 & 3.9 \\
AFL & 78.6 & 11.4 & 4.0 \\
TCC & 75.8 & 24.3 & 4.4 \\
tVC & 68.5 & 10.8 & 5.4 \\
OTA & 63.3 & 1.3 & 6.1 \\
Peel & 47.1 & 0.0 & 8.4 \\
AA & 46.3 & 3.5 & 8.5 \\
MN & 45.6 & 1.1 & 8.6 \\
tTA & 33.2 & 0.0 & 10.4 \\
Tretinoin & 32.9 & 0.4 & 10.4 \\
PICO & 32.8 & 0.5 & 10.4 \\
HQ & 29.4 & 0.0 & 10.9 \\
NAFL & 28.1 & 0.5 & 11.1 \\
Placebo & 3.6 & 0.0 & 14.5 \\
\hline
\end{tabular}

*SUCRA: Surface under the cumulative ranking curve. The higher the SUCRA score, the better the treatment; PrBest indicates the possibility that the treatment is the best treatment.

$A A$, azelaic acid; $A F L$, ablative fractional laser; $H Q$, hydroquinone; IPL, intense pulsed light; MN, microneedling; NAFL, nonablative fractional laser; OTA, oral tranexamic acid; PICO, picosecond laser; QSND, Q-switched Nd:Yag 1,064-nm laser; TCC, triple combination cream; tTA, topical tranexamic acid; $t V C$, topical vitamin $C$.
In addition to the inhibition of melanogenesis or destruction of melanin granule, the inhibitory effect of steroids and TA on ET-1 (endothelin 1), the improvement effect of TA on blood

TABLE 2 | Summary of side effects.

\begin{tabular}{lccc}
\hline Treatment & $\begin{array}{c}\boldsymbol{N} \text { (total number of } \\
\text { patients) }\end{array}$ & $\begin{array}{c}\boldsymbol{N} \text { (number of } \\
\text { patients with SE) }\end{array}$ & $\mathbf{n / N} \%$ \\
\hline IPL & 47 & 0 & 0.0 \\
Tretinoin & $\mathbf{1 0 8}$ & 11 & 10.1 \\
tVC & 31 & 5 & 16.1 \\
oTA & $\mathbf{2 5 5}$ & 45 & 17.6 \\
HQ & $\mathbf{4 3 4}$ & 79 & 18.2 \\
AFL & $\mathbf{1 5 5}$ & 31 & 20.0 \\
QSND & $\mathbf{2 9 3}$ & 63 & 21.5 \\
AA & 64 & 15 & 23.4 \\
TCC & $\mathbf{9 7}$ & 25 & 25.7 \\
tTA & $\mathbf{3 0 2}$ & 111 & 36.7 \\
Peel & $\mathbf{2 1 3}$ & 81 & 38.0 \\
PICO & 20 & 9 & 45.0 \\
MN & $\mathbf{8 6}$ & 45 & 52.3 \\
NAFL & 27 & 18 & 66.6 \\
\hline
\end{tabular}

*Only the reported side effects in monotherapy studies are listed. Monotherapies with more than 80 participants are highlighted in italic boldface. The remaining monotherapies below 80 participants are in normal font.

$A A$, azelaic acid; $A F L$, ablative fractional laser; $H Q$, hydroquinone; IPL, intense pulsed light; MN, microneedling; NAFL, nonablative fractional laser; OTA, oral tranexamic acid; PICO, picosecond laser; QSND, Q-switched Nd:Yag 1,064-nm laser; SE, side effect; TCC, triple combination cream; tTA, topical tranexamic acid; tVC, topical vitamin C.

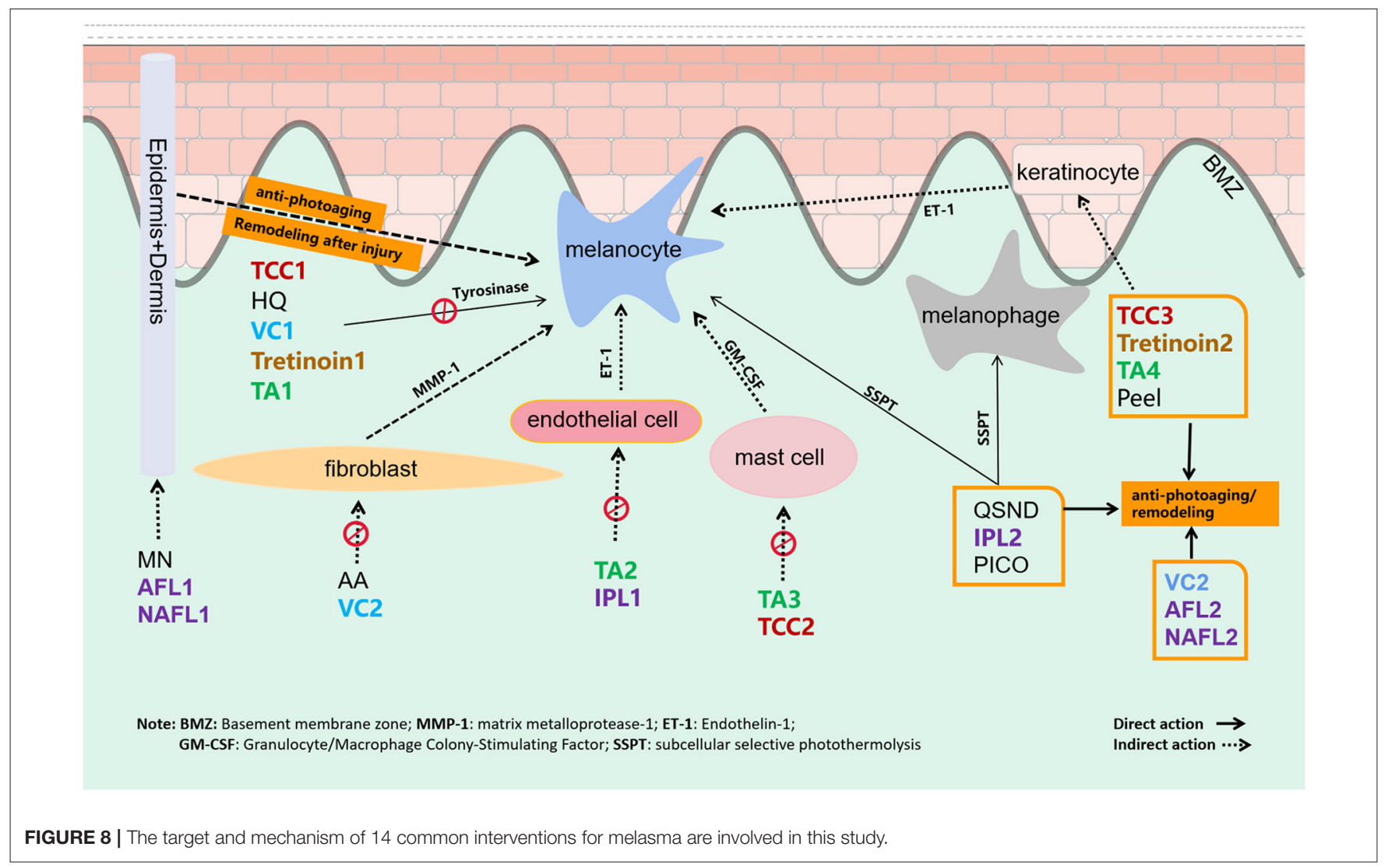


vessels and mast cells, and the effect of the laser by reversing the characteristics of photoaging on melasma also should be taken into account. These therapeutic targets seem to play an important role in the treatment of melasma. The effect of combination therapy with multiple targets among drugs, energybased devices or $\mathrm{MN}$, and so on would be better than that of single therapy, and it is possible to reduce the recurrence of melasma.

This study was restricted by the limited number of RCTs, particularly those related to PICO laser. Hence, it is necessary to perform more RCTs and split-face studies that can reduce the $\mathrm{ROB}$ to update the current results and help develop more standardized protocols. These data may direct doctors to better treat patients with melasma. In clinical application, more attention needs to be paid to the efficacy and safety of the treatment. Based on this, the combination therapy of multiple targets on mechanism may produce unexpected effects. As for the ranking of melasma monotherapy, we believe that following up the release of new trials, the network will expand, and the ranking list may change a little.

\section{REFERENCES}

1. Grimes PE. Melasma. Etiologic and therapeutic considerations. Arch Dermatol. (1995) 131:1453-7. doi: 10.1001/archderm.131.12.1453

2. Werlinger KD, Guevara IL, González CM, Rincón ET, Caetano R, Haley RW, et al. Prevalence of self-diagnosed melasma among premenopausal Latino women in Dallas and Fort Worth, Tex. Arch Dermatol. (2007) 143:4245. doi: 10.1001/archderm.143.3.424

3. Rodrigues M, Pandya AG. Melasma: clinical diagnosis and management options. Australas J Dermatol. (2015) 56:151-63. doi: 10.1111/ajd.12290

4. Ponzio HA, Favaretto AL, Rivitti EA. Proposal of a quantitative method to describe melasma distribution in women. J Cosmet Dermatol. (2007) 20:10311.

5. Handel AC, Miot LD, Miot HA. Melasma: a clinical and epidemiological review. An Bras Dermatol. (2014) 89:77182. doi: 10.1590/abd1806-4841.20143063

6. Kwon SH, Na JI, Choi JY, Park KC. Melasma: Updates and perspectives. Exp Dermatol. (2019) 28:704-8. doi: 10.1111/exd.13844

7. Sanchez NP, Pathak MA, Sato S, Fitzpatrick TB, Sanchez JL, Mihm MC. Melasma: a clinical, light microscopic, ultrastructural, and immunofluorescence study. $J$ Am Acad Dermatol. (1981) 4:698-710. doi: 10.1016/S0190-9622(81)70 071-9

8. Sirithanabadeekul P, Dannarongchai A, Suwanchinda A. Platelet-rich plasma treatment for melasma: A pilot study. J Cosmet Dermatol. (2020) 19:13217. doi: 10.1111/jocd.13157

9. Austin E, Nguyen JK, Jagdeo J. Topical treatments for melasma: A systematic review of randomized controlled trials. J Drugs Dermatol. (2019). 18:S1545961619P1156X.

10. Rivas S, Pandya AG. Treatment of melasma with topical agents, peelings and lasers: an evidence-based review. Am J Clin Dermatol. (2013) 14:359-76. doi: 10.1007/s40257-0130038-4

11. Spierings NMK. Melasma: A critical analysis of clinical trials investigating treatment modalities published in the past 10 years. J Cosmet Dermatol. (2020) 19:1284-9. doi: 10.1111/jocd.13182

12. Park KY, Kim DH, Kim HK Li K, Seo SJ, Hong CK, A. randomized, observerblinded, comparison of combined 1064-nm Q-switched neodymium-doped yttrium-aluminum-garnet laser plus $30 \%$ glycolic acid peeling vs. laser

\section{DATA AVAILABILITY STATEMENT}

The original contributions presented in the study are included in the article/Supplementary Material, further inquiries can be directed to the corresponding author.

\section{AUTHOR CONTRIBUTIONS}

YL contributed to data collection and article writing. SW contributed to statistical methods. HW participated in the article screening. XL participated in the chart making. DG participated in the article revision, and the article topic selection and final revision were in the charge of FZ. All authors contributed to the article and approved the submitted version.

\section{SUPPLEMENTARY MATERIAL}

The Supplementary Material for this article can be found online at: https://www.frontiersin.org/articles/10.3389/fmed. 2021.713554/full\#supplementary-material monotherapy to treat melisma. J Clin Exp Dermatol Res. (2011) 36:86470. doi: 10.1111/j.1365-2230.2011.04150.x

13. Nouri K, Bowes L, Chartier T, Romagosa R, Spencer J. Combination treatment of melasma with pulsed $\mathrm{CO} 2$ laser followed by Qswitched alexandrite laser: a pilot study. Dermatol Surg. (1999) 25:494-7. doi: 10.1046/j.1524-4725.1999.08248.x

14. Hutton B, Salanti G, Caldwell DM, Chaimani A, Schmid CH, Cameron C, et al. The PRISMA extension statement for reporting of systematic reviews incorporating network meta -analyses of health care interventions: checklist and explanations. Ann Intern Med. (2015) 162:777-84. doi: 10.7326/M14-2385

15. Higgins JPT, Green S. Cochrane Handbook for Systematic Reviews of Interventions Version 5.1.0. (2011). Available online at: http://handbook. cochrane.org/ (accessed April 27, 2016).

16. Salanti G. Indirect and mixed-treatment comparison, network, or multiple treatments meta-analysis: Many names, many benefits, many concerns for the next generation evidence synthesis tool. Res Synth Methods. (2012) 3:8097. doi: 10.1002/jrsm.1037

17. Higgins JP, Jackson D, Barrett JK, Lu G, Ades AE, White IR. Consistency and inconsistency in networkmeta-analysis: concepts and models for multi-arm studies. Res Synth Methods. (2012) 3:98-110. doi: 10.1002/jrsm.1044

18. Mbuagbaw L, Rochwerg B, Jaeschke R, Heels-Andsell D, Alhazzani W, Thabane L, et al. Approaches to interpreting and choosing the best treatments in network meta-analyses. Syst Rev. (2017) 6:79. doi: 10.1186/s13643-017-0473-z

19. Kim JH, Kim H, Park HC, Kim IH. Subcellular selective photothermolysis of melanosomes in adult zebrafish skin following 1064-nm Qswitched Nd:YAG laser irradiation. J Invest Dermatol. (2010) 130:2333-5. doi: 10.1038/jid.2010.129

20. DiBernardo BE, Pozner JN. Intense pulsed light therapy for skin rejuvenation. Clin Plast Surg. (2016) 43:535-40. doi: 10.1016/j.cps.2016.03.008

21. Sheth VM, Pandya AG. Melasma: a comprehensive update: part II. J Am Acad Dermatol. (2011) 65:699-714. doi: 10.1016/j.jaad.2011.06.001

22. Manaloto RM, Alster T. Erbium: YAG laser resurfacing for refractory melasma. Dermatol Surg. (1999) 25:1213. doi: 10.1046/j.1524-4725.1999.08103.x

23. El-Sinbawy Zenab G, Abdelnabi N M, Sarhan N E, Elgarhy LH. Clinical \& ultrastructural evaluation of the effect of fractional CO2 laser on facial melisma. Ultrastruct Pathol. (2019) 43:13544. doi: 10.1080/01913123.2019.1673861 
24. Sim JH, Park YL, Lee JS, Lee SY, Choi WB, Kim HJ, et al. Treatment of melasma by low-fluence $1064 \mathrm{~nm}$ Q-switched Nd:YAG laser. J Dermatolog Treat. (2014) 25:212-7. doi: 10.3109/09546634.2012.7 35639

25. Kaminaka C, Furukawa F, Yamamoto Y. The clinical and histological effect of a low-fluence Q-switched 1,064-nm neodymium: yttriumaluminum-garnet laser for the treatment of melasma and solar lentigenes in asians: prospective, randomized, and split-face comparative study. Dermatol Surg. (2017) 43:1120-33. doi: 10.1097/DSS.00000000000 01120

26. Choi JE, Lee DW, Seo SH, Ahn HH, Kye YC. Low-fluence Q-switched Nd:YAG laser for the treatment of melasma in Asian patients. J Cosmet Dermatol. (2018) 17:1053-8. doi: 10.1111/jocd.12760

27. Abdel HR, Mohammed FN, Sayed KS, Abd EI Fattah NA, Ibrahim S. Dermoscopy as a useful tool for evaluating melasma and assessing the response to 1064-nm Q-switched Nd:YAG laser. Dermatol Ther. (2020) 33:e13629. doi: 10.1111/dth.1 3629

28. Longo C, Pellacani G, Tourlaki A, Galimberti M, Bencini PL. Melasma and low-energy Q-switched laser: treatment assessment by means of in vivo confocal microscopy. Lasers Med Sci. (2014) 29:1159-63. doi: 10.1007/s10103-013-1498-8

29. Yi J, Hong T, Zeng H, Li P, Li P, Wang S, et al. A meta-analysis based assessment of intense pulsed light for treatment of melasma. Aesthetic Plast Surg. (2020) 44:947-52. doi: 10.1007/s00266-020-01637-x

30. Wanitphakdeedecha R, Manuskiatti W, Siriphukpong S, Chen TM. Treatment of melasma using variable square pulse Er:YAG laser resurfacing. Dermatol Surg. (2009). 35:475-81. doi: 10.1111/j.1524-4725.2009.01066.x

31. Badawi AM, Osman MA. Fractional erbium-doped yttrium aluminum garnet laser-assisted drug delivery of hydroquinone in the treatment of melasma. Clin Cosmet Investig Dermatol. (2018). 11:13-20. doi: 10.2147/CCID.S147413

32. Farshi S. Comparative study of therapeutic effects of $20 \%$ azelaic acid and hydroquinone $4 \%$ cream in the treatment of melasma. J Cosmet Dermatol. (2011) 0:282-7. doi: 10.1111/j.1473-2165.2011.00580.x

33. Lee MC, Lin YF, Hu S, Huang YL, Chang SL, Cheng CY, et al. A split-face study: comparison of picosecond alexandrite laser and Q-switched Nd:YAG laser in the treatment of melasma in Asians. Lasers Med Sci. (2018) 33:17338. doi: 10.1007/s10103-018-2529-2

34. Jo DJ, Kang IH, Baek JH, Gwak MJ, Lee SJ, Shin MK. Using reflectance confocal microscopy to observe in vivo melanolysis after treatment with the picosecond alexandrite laser and Q-switched Nd:YAG laser in melasma. Lasers Surg Med. (2018) 111:40-8. doi: 10.1002/lsm.23025

35. Kligman AM, Willis I. A new formula for depigmenting human skin. Arch Dermatol. (1975) 111:40-8. doi: 10.1001/archderm.111.1.40

36. McKesey J, Tovar-Garza A, Pandya AG. Melasma treatment: an evidence-based review. Am J Clin Dermatol. (2020) 21:173225. doi: 10.1007/s40257-019-00488-w

37. Taylor SC, Torok H, Jones T, Lowe N, Rich P, Tschen E, et al. Efficacy and safety of a new triplecombination agent for the treatment of facial melasma. Cutis. (2003) 72:67-72. doi: 10.1016/S1040-0486(03)00017-6

38. Ferreira CT, Hassun K, Sittart A, Lourdes VM, A. comparison of triple combination cream and hydroquinone $4 \%$ cream for the treatment of moderate to severe facial melasma. J Cosmet Dermatol. (2007) 6:369. doi: 10.1111/j.1473-2165.2007.00288.x

39. Chan R, Park KC, Lee MH, Lee ES, Chang SE, Leow YH. et al. A randomized controlled trial of the efficacy and safety of a fixed triple combination (fluocinolone acetonide 001\%, hydroquinone $4 \%$, tretinoin 005\%) compared with hydroquinone $4 \%$ cream in Asian patients with moderate to severe melisma. Br J Dermatol. (2008) 159:697703. doi: 10.1111/j.1365-2133.2008.08717.x

40. Wang YJ, Lin ET, Chen YT, Chiu PC, Lin BS, Chiang HM, et al. Prospective randomized controlled trial comparing treatment efficacy and tolerance of picosecond alexandrite laser with a diffractive lens array and triple combination cream in female Asian patients with melasma. J Eur Acad Dermatol Venereol. (2020) 34:624-32. doi: 10.1111/jdv.15934
41. Saki N, Darayesh M, Heiran A. Comparing the efficacy of topical hydroquinone $2 \%$ versus intradermal tranexamic acid microinjections in treating melasma: a split-face controlled trial. J Dermatolog Treat. (2018) 29:405-10. doi: 10.1080/09546634.2017.1392476

42. Farris PK. Topical vitamin C: A useful agent for treating photoaging and other dermatologic conditions. Dermatol Surg. (2005) 31:814-7. doi: 10.1111/j.1524-4725.2005.31725

43. Na JI, Choi SY, Yang SH, Choi HR, Kang HY, Park KC. Effect of tranexamic acid on melasma: a clinical trial with histological evaluation. J Eur Acad Dermatol Venereol. (2013) 27:1035-9. doi: 10.1111/j.1468-3083.2012.04464.x

44. Kim SJ, Park JY, Shibata T, Fujiwara R, Kang HY. Efficacy and possible mechanisms of topical tranexamic acid in melasma. Clin Exp Dermatol. (2016) 41:480-5. doi: 10.1111/ced.12835

45. Wang JV, Jhawar N, Saedi N. Tranexamic Acid for Melasma: Evaluating the Various Formulations. J Clin Aesthet Dermatol. (2019) 12:E73-4.

46. Chee-Leok G, Sai Yee C, Thng Guan TS, Alejandra VM, Arancha DR. Polypodium leucotomos double-blind, placebo-controlled trial to evaluate the effectiveness of extract in the treatment of melasma in asian skin: a pilot study. J Clin Aesthet Dermatol. (2018). 11:14-19.

47. Ahmed Ammar M. Lopez Isha, Perese Francisco, Vasquez Rebecca, Hynan Linda S, Chong Benjamin, et al. A randomized, doubleblinded, placebo-controlled trial of oral Polypodium leucotomos extract as an adjunct to sunscreen in the treatment of melisma. JAMA Dermatol. (2013) 149:981-3. doi: 10.1001/jamadermatol.201 3.4294

48. Sarkar R, Arsiwala S, Dubey N. Sonthalia Sidharth, Das Anupam, Arya Latika, et al. Chemical Peels in Melasma: A Review with Consensus Recommendations by Indian Pigmentary Expert Group. Indian J Dermatol. (2017) 62:578-84. doi: 10.4103/ijd.IJD_490_17

49. Dorgham NA, Hegazy RA, Sharobim AK, Dorgham DA. Efficacy and tolerability of chemical peelinging as a single agent for melasma in dark-skinned patients: A systematic review and meta-analysis of comparative trials. J Cosmet Dermatol. (2020) 19:2812-9. doi: 10.1111/jocd. 13725

50. Cassiano DP, Espósito ACC, Hassun KM, Lima EVA, Bagatin E, Miot HA. Early clinical and histological changes induced by microneedling in facial melasma: A pilot study. Indian J Dermatol Venereol Leprol. (2019) 85:63841. doi: 10.4103/ijdvl.IJDVL_44_19

51. Sarma N, Chakraborty S, Poojary SA, Rathi S, Kumaran S, Nirmal B, et al. Evidence-based review, grade of recommendation, and suggested treatment recommendations for melasma. Indian Dermatol Online J. (2017) 8:40642. doi: 10.4103/idoj.IDOJ_187_17

52. Neagu N, Conforti C, Agozzino M, Marangi GF, Morariu SH, Pellacani G, et al. Melasma treatment: a systematic review. J Dermatolog Treat. (2021) 14:1-39. doi: 10.1080/09546634.2021.1914313

Conflict of Interest: The authors declare that the research was conducted in the absence of any commercial or financial relationships that could be construed as a potential conflict of interest.

Publisher's Note: All claims expressed in this article are solely those of the authors and do not necessarily represent those of their affiliated organizations, or those of the publisher, the editors and the reviewers. Any product that may be evaluated in this article, or claim that may be made by its manufacturer, is not guaranteed or endorsed by the publisher.

Copyright (c) $2021 \mathrm{Liu}, W u, W u$, Liang, Guo and Zhuo. This is an open-access article distributed under the terms of the Creative Commons Attribution License (CC $B Y)$. The use, distribution or reproduction in other forums is permitted, provided the original author(s) and the copyright owner(s) are credited and that the original publication in this journal is cited, in accordance with accepted academic practice. No use, distribution or reproduction is permitted which does not comply with these terms. 